\title{
PREFACE: UKRAINIAN SPECIAL ISSUE OF FLUID MECHANICS RESEARCH
}

Dear readers, with excitement and joy, I present the content of a special issue of our Journal dedicated to the recent achievements of the Institute of Hydromechanics of the National Academy of Sciences of Ukraine, the leading national research center in the fields of Fluid Mechanics, Hydraulic Engineering and Acoustics. Nowadays, we are faced with demands to enhance the global visibility of Ukrainian scientists, so another opportunity to take the floor in a respected foreign scientific Journal is very important for us. It is worth noting that most of the results presented here were obtained in the course of international research projects.

This issue is a product of the long-term cooperation between our Institute and Begell House, Inc. The incredible work on the selection of publications could not have been done without the comprehensive assistance of the Director of our Institute, Academician of the National Academy of Sciences of Ukraine, Prof. Victor Grinchenko, who until recently was the Editor-in-Chief of the International Journal of Fluid Mechanics Research. We are deeply grateful to our partners for the creation of an atmosphere of goodwill, mutual understanding, respect and trust.

The issue is started with the paper by Vladimir Nikishov, Tetiana Schcherbak and Natalia Gorodetska studying the scattering of surface gravitational waves in an incompressible liquid on a submerged barrier. Such problems are of certain interest for the design of structures for coastal protection; special attention should be paid to the methodological value of the approach proposed here. Within the linearized approximation for small-amplitude waves, the problem is solved by the method of partial domains that has been successfully applied for decades to wave problems of Acoustics and Solid-State Mechanics. Its essence consists in formal separation of the entire complex domain of the field existence into subdomains, each of which possesses a canonical geometry that enables to write the corresponding general solution in the form of a series with respect to a priori known eigenfunctions. This work is significant precisely because it widens the range of problems that can be solved by the method of partial domains. We also dedicate this publication to the blessed memory of our colleague Prof. Vladimir Nikishov, a remarkable scientist and person who has left us one year ago.

The experimental and theoretical study of hydrodynamic cavitation is one of the calling cards of the Institute of Hydromechanics. Our results of the research of high-speed motion of cavitating flooded bodies are deservedly recognized abroad. When organizing such flow regimes, one of the biggest challenges is to provide the stability and controllability of the object moving in the supercavity. The ability of high-speed cavitating vehicles for threedimensional maneuvering is the central problem of the paper by Yurii Savchenko, Vladimir Semenenko and Georgii Savchenko.

Unlike the case of supercavitation when drag reduction is achieved due to a significant decrease of the wetted body surface in the continuous flow regimes, the quality of the boundary layer in the adjacent fluid plays a predominant role. It is known that it can yield both positive and negative effects. An interesting technique for controlling the wall vorticity structure, which was borrowed from the nature itself, is the use of deformable sections of a streamlined surface. The paper by Gennadii Voropaiev and Iaroslav Zagumennyi deals with the estimation of the effect of elastically deformable insert on the development of wall coherent vortex structures.

The paper written by a large Ukrainian-Italian team under the command of Vladimir Voskoboinick illustrates the systematic interest of our Institute to Biomedical problematics. Here, the hydrodynamic noise from open and semiopen artificial prosthetic mitral valve is studied. As in the previous case, the discussed acoustical effects are generated by the boundary layer vortex structures and can serve as an important diagnostic marker in assessing the operation of cardiac implants.

In modern science, it is often important not only in the depth of immersion into a problem, but also the breadth of the vision that makes it possible to reveal similar features in seemingly remote phenomena. Nina Yurchenko's 
paper successfully demonstrates the fruitfulness of such an interdisciplinary approach to the problems of boundary layer control in such diverse areas as Aerodynamics and Bionics. The undoubted beauty of the results and the artistic presentation of the data make this material interesting not only for scientific communities, but also for the general public.

I also would like to thank Begell House, Inc., for the honor given to me to act as a guest editor of this special issue of the Journal. In my opinion, its content will be of interest for the researchers focusing their efforts on Fluid, Gas and Plasma Mechanics, and our meetings in this format will become regular.

My special thanks to the language editor of this issue, Nataliia Matsypura, whose work has been an important contribution to the improvement of the articles' presentation.

Valery Oliynik,

Deputy Editor of the Journal 